comorbid substance use in observational studies and clinical practice.

\section{The impact of accommodation status on the hospitalization of mentally ill patients}

\author{
A Bonett', S Tahtalian ${ }^{2,3}$, B Hayward' ', J Kulkarni' ${ }^{2,3}$, \\ Y Hollander ${ }^{1}$ \\ 'Alfred Hospital Psychiatry Department; ${ }^{2}$ Alfred Psychiatry Research Centre; and \\ ${ }^{3}$ Monash University, Melbourne, Australia
}

Background: The importance of suitable and stable housing has long been recognized for people suffering mental illness. In recent years, there has been a steady decrease in the availability of housing options for this group of people. Anecdotal evidence suggests that this is now impacting on their length of hospitalization and health outcomes.

Method: Patients were enrolled over a 3-month period during their admission to the Alfred Hospital psychiatric ward. In-patient social workers collected information for every consecutive admission regarding patient's current accommodation situation and their attempt to secure appropriate housing for these patients. Demographic information was also collected. Consultant psychiatrists measured symptom severity using the Brief Psychiatric Rating Scale.

Results: Data presented will include a summary of the appropriateness of the current housing situation of participants at admission, the accommodation options available for discharge, the time taken to secure the accommodation, the time spent in hospital after medical clearance for discharge and the length of hospitalization. An analysis will assess whether patients who are considered to be in unstable accommodation are experiencing longer hospital stays as compared with those in stable housing.

Conclusions: The results will examine how the decreases in accommodation options are impacting on the use of hospital resources and patient outcomes. This information is imperative given the limited inpatient psychiatric resources available.

\section{Keep the heart in mind: the interplay of heart and brain activity over the life span}

\section{P Boord', L Williams', E Gordon², C Rennie'}

'Brain Dynamics Centre, Westmead Millenium Institute, Westmead Hospital and the University of Sydney, Australia; and 'Brain Resource Company, Sydney, Australia
Background: Affective disorders are associated with both visceral and neurophysiological changes, but few studies report simultaneous measurement and analysis of these systems. We used an integrative neuroscience approach to explore relationships between heart and brain activity for healthy controls, as a benchmark for studying the interplay of these systems in affective disorders.

Methods: Participants were recruited in collaboration with the Brain Resource International Database (www. brainresource.com). Simultaneous EEG and ECG were recorded from 2092 healthy individuals while they rested with their eyes open. EEG power was calculated in standard frequency bands, averaged across sites and correlated with average heart rate for each decade of the life span.

Results: Heart rate was significantly correlated with EEG power in young people (10-20 years old) but was less evident in people outside this age range. In this age group, heart rate and EEG power had a positive correlation across all frequency bands (delta, theta, alpha, beta $r=+0.25,+0.20,+0.14,+0.19$, respectively; $n=675, P<0.001$ ).

Conclusions: This study shows the imperative of using an integrative neuroscience approach in the study of brain function and dysfunction. Without taking account of the manifold influences on brain function, neurophysiological studies might be confounded by variance in these factors. Simultaneous measurement across systems can also disclose their interaction in health and disease. The observed change in interplay between the heart and brain over age might prove an important factor in the understanding of affective disorders.

\section{Reduced fMRI activity in response to salient stimuli in first-episode schizophrenia}

\section{K Brown', D Alexander², P Boord', P Das 1,3, G Flynn', C Galletly ${ }^{5}$, E Gordon ${ }^{1,2}$, A Harris ${ }^{1,6}$, TJ Whitford', W Wong ${ }^{4}$, L Williams ${ }^{1,6}$}

'Brain Dynamics Centre, Westmead Millenium Institute, Westmead Hospital and the University of Sydney, Australia; ${ }^{2 B r a i n}$ Resource Company; ${ }^{3}$ Neuroscience Institute of Schizophrenia and Allied Disorders (NISAD); "Liverpool Hospital, Early Psychosis Intervention Program; ${ }^{5}$ University of Adelaide; and ${ }^{6}$ The University of Sydney, Australia

Background: Our integrative neuroscience model of schizophrenia highlights the lack of coordinated neural activity required for effective processing of salient and task-relevant stimuli. The 'auditory oddball' task taps the fundamental mechanisms of selecting and responding to salient stimuli, and disturbances on this task are a trait-like marker for schizophrenia. The objective of 